\title{
Survival with first-line bosentan in patients with primary pulmonary hypertension
}

\author{
V.V. McLaughlin*, O. Sitbon" ${ }^{\#}$ D.B. Badesch ${ }^{\star}$, R.J. Barst ${ }^{+}$, C. Black ${ }^{\S}$, N. Galiè ${ }^{f}$, \\ M. Rainisio**, G. Simonneau ${ }^{\#}$ and L.J. Rubin ${ }^{\# \#}$
}

ABSTRACT: Primary pulmonary hypertension (PPH) is a progressive disease with high mortality. Administration of i.v. epoprostenol has demonstrated improved exercise tolerance, haemodynamics, and survival. The orally active, dual endothelin receptor antagonist bosentan improves exercise endurance, haemodynamics, and functional class over the short term. To determine the effect of first-line bosentan therapy on survival, this study followed 169 patients with PPH treated with bosentan in two placebo-controlled trials and their extensions.

Data on survival and alternative treatments were collected from September 1999 (start of the first placebo-controlled study) to December 31, 2002. Observed survival up to 36 months was reported as Kaplan-Meier estimates and compared with predicted survival as determined for each patient by the National Institutes of Health Registry formula.

Kaplan-Meier survival estimates were $96 \%$ at 12 months and $89 \%$ at 24 months. In contrast, predicted survival was $69 \%$ and $57 \%$, respectively. In addition, at the end of 12 and 24 months, $85 \%$ and $70 \%$ of patients, respectively, remained alive and on bosentan monotherapy. Factors that predicted a worse outcome included World Health Organization Functional Class IV and 6-min walk distance below the median $(358 \mathrm{~m})$ at baseline.

First-line bosentan therapy was found to improve survival in patients with advanced primary pulmonary hypertension.

KEYWORDS: Bosentan, endothelin receptor antagonists, primary pulmonary hypertension, pulmonary hypertension, survival

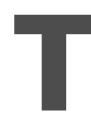
he treatment of primary pulmonary hypertension (PPH), historically viewed as a progressive and fatal disease, has significantly improved over the past decade. Observational studies have demonstrated a mortality benefit in PPH with warfarin anticoagulation $[1,2]$, while calcium channel blockers improve survival in a small subset of $\mathrm{PPH}$ patients who demonstrate marked vasoreactivity [2]. In a 12week randomised, open-label trial of 81 functional class III and IV PPH patients, a survival benefit was demonstrated in patients treated with i.v. epoprostenol compared with patients treated with conventional therapy [3]. More recently, two large observational studies have confirmed the longterm mortality benefit among functional class III and IV PPH patients treated with epoprostenol when compared with either historical controls or predicted survival based on the National Institutes of Health (NIH) Registry equation, which incorporates right atrial pressure, cardiac index and mean pulmonary artery pressure $[4,5]$. While

For editorial comments see page 218. epoprostenol improves exercise tolerance, haemodynamics, and survival, its use is limited by its cumbersome delivery system, which requires continuous i.v. infusion. Deleterious effects, including infection and infusion interruption, may be life threatening.

The dual endothelin receptor antagonist bosentan (Ro 47-0203; Tracleer ${ }^{\circledR}$, Actelion Pharmaceuticals, Allschwil, Switzerland) is the first oral therapy approved for the treatment of pulmonary arterial hypertension (PAH). Among patients with either $\mathrm{PPH}$ or PAH related to the scleroderma spectrum of disease, randomised, double-blind, placebocontrolled studies have demonstrated that bosentan therapy improves haemodynamics, exercise capacity, and functional class [6, 7]. In addition, a delay in time to clinical worsening was demonstrated in bosentan-treated patients compared with placebo, with clinical benefits maintained for up to 28 weeks [7]. In a long-term, open-label extension study, the improvement in functional class persisted for up to $1 \mathrm{yr}$ [8].

Patients who participated in these studies have now received bosentan as first-line therapy for up
AFFILIATIONS

*University of Michigan, Ann Arbor, MI, USA.

\#University Paris-Sud, Clamart, France.

-University of Colorado Health Sciences Center, Denver, C0, USA ${ }^{+}$Columbia University College of Physicians and Surgeons, New York, NY, USA.

${ }^{\S}$ Royal College of Physicians, London, UK.

fUniversity of Bologna, Bologna, Italy.

**Actelion Pharmaceuticals Ltd, Allschwil, Switzerland.

\#\#University of California, San Diego, CA, USA.

CORRESPONDENCE

V.V. McLaughlin

University of Michigan

1500 East Medical Center Drive

Women's Hospital, Room L3119

Ann Arbor

$\mathrm{MI}$

USA

Fax: 17347637390

E-mail: vmclaugh@med.umich.edu

Received:

May 072004

Accepted after revision:

October 042004

\section{SUPPORT STATEMENT}

This study was supported by a grant from Actelion Pharmaceuticals Ltd., Allschwil, Switzerland. 
to 3 yrs. The objective of this study was to evaluate the effect of a treatment strategy beginning with first-line bosentan therapy, followed by the addition of other therapies if needed, on survival in $\mathrm{PPH}$ patients compared with their predicted survival, as determined by the formula of D'ALONZO et al. [9] based on NIH Registry data [10]. In addition, the use of other targeted treatments, outcomes, and prognostic factors for survival in bosentan-treated PPH patients were explored.

\section{METHODS \\ Patients}

Patients in the analyses were enrolled in the two placebocontrolled trials of bosentan in PAH [6, 7]. At entry, patients had severe symptomatic (World Health Organization Functional Class III or IV) PAH, either primary or associated with connective tissue disease. Additional entry criteria included: age $\geqslant 12$ yrs, mean pulmonary arterial pressure $>25 \mathrm{mmHg}$, pulmonary vascular resistance $>3 \mathrm{mmHg} \cdot \mathrm{L}^{-1} \cdot \mathrm{min}^{-1}$, and pulmonary capillary wedge pressure $<15 \mathrm{mmHg}$ as measured by right heart catheterisation, and 6-min walk distance (6MWD) of $150-450 \mathrm{~m}$ at baseline. Patients had no previous exposure to prostanoids, and background therapy was based on the discretion of the treating physician. Patients who completed a placebo-controlled study were eligible to continue or start bosentan treatment in the open-label extension study. The target dose of bosentan in the first placebo-controlled trial was $125 \mathrm{mg}$ twice daily. In the second placebo-controlled trial, patients were randomised in a 1:1:1 fashion to placebo, a target dose of $125 \mathrm{mg}$ twice daily, or a target dose of $250 \mathrm{mg}$ twice daily. During the open-label extensions, the target dose was $125 \mathrm{mg}$ twice daily with the option to up-titrate to $250 \mathrm{mg}$ twice daily in cases of clinical deterioration. All studies were conducted in accordance with the amended Declaration of Helsinki at sites in North America, Europe, Australia, and Israel. Studies were approved by a local ethics review committee, and written informed consent was obtained from all patients.

Of the 245 patients enrolled in the placebo-controlled studies, $\mathrm{PAH}$ was considered primary in 177, and analyses were performed in the $169 \mathrm{PPH}$ patients who received bosentan as first-line therapy for their disease, either during the placebocontrolled study or its extension. Eight patients initially randomised to placebo, seven of whom were withdrawn and treated with alternative therapies and one who died before the end of the placebo-controlled trial, did not receive bosentan as first-line therapy and were excluded from the analysis. Decisions on other treatments were up to the treating physician. During the placebo-controlled studies, patients requiring prostanoid therapy were withdrawn from the study; during the extension studies, prostanoid or other alternate oral therapies could be administered with continued bosentan therapy. Data on vital status and alternative treatments were collected from September 1999 (start of the first placebocontrolled study) to December 31, 2002 (data cut-off), whether or not patients remained on study treatment throughout.

\section{Statistical analyses}

Baseline and follow-up information were summarised as mean $\pm \mathrm{SD}$ or frequency counts and proportions. Baseline parameters were recorded at the start of bosentan treatment where possible.
Survival was assessed from the start of bosentan treatment to death or data cut-off. All bosentan-treated PPH patients were included in the analyses (intent to treat); patients lost to followup were considered dead at the last known contact. Changes in treatment did not affect the survival analysis. Kaplan-Meier estimates up to 36 months are reported with $99.9 \%$ confidence intervals. The annual death rate was obtained by interpolation of the observed data assuming an exponential distribution.

Expected survival was calculated for each patient based on the NIH formula:

$$
\mathrm{P}(\mathrm{t})=[\mathrm{H}(\mathrm{t})]^{\mathrm{A}(\mathrm{x}, \mathrm{y}, \mathrm{z})}
$$

where $\mathrm{H}(\mathrm{t})=0.88-0.14 \mathrm{t}+0.01 \mathrm{t}^{2}$ ( $\mathrm{t}$ being the time in years), $\mathrm{A}(\mathrm{x}, \mathrm{y}, \mathrm{z})=\mathrm{EXP}(0.007325 \mathrm{x}+0.0526 \mathrm{y}-0.3275 \mathrm{z}), \mathrm{x}$ is mean pulmonary artery pressure, $\mathrm{y}$ is mean right atrial pressure, and $\mathrm{z}$ is cardiac index. The probabilities of survival at 1,2 , and 3 yrs are given by the following: $\mathrm{P}(1)=0.75^{\mathrm{A}} ; \mathrm{P}(2)=0.64^{\mathrm{A}}$; and $\mathrm{P}(3)=0.55^{\mathrm{A}}[9]$. The formula was based on data from $187 \mathrm{PPH}$ patients in the NIH Registry [10]; this prediction equation has been validated prospectively in $61 \mathrm{PPH}$ patients [11].

Vital status and treatment at 12 and 24 months were summarised as the proportions of patients who completed 12 and 24 months, respectively. Patients lost to follow-up or with missing information were counted as dead or off bosentan therapy, respectively, at the time of the last available information. Prognostic factors were explored by univariate analysis using the Cox proportionalhazards model; hazard ratios with $95 \%$ confidence intervals were reported. The incidence of adverse events and Kaplan-Meier estimates of time to first occurrence of liver transaminase elevation to greater than three times the upper limit of normal were reported for the adverse event observation periods of the four clinical studies.

\section{RESULTS}

Baseline characteristics of the 169 bosentan-treated PPH patients are displayed in table 1. Although not directly used in the analysis, available data from the NIH Registry of PPH patients are also shown. Patients in the bosentan database were representative of the $\mathrm{PPH}$ patient population in the $\mathrm{NIH}$ Registry. Although the NIH Registry contained more class II patients than were in the bosentan studies, haemodynamics were similar in both groups. At the time of the last observation, the majority $(77.4 \%)$ of patients on bosentan were taking bosentan $125 \mathrm{mg}$ twice per day. Altogether, $1 \%$ of patients were taking $62.5 \mathrm{mg}$ per day, $11.3 \%$ were taking $62.5 \mathrm{mg}$ twice per day, and $10.1 \%$ were taking $250 \mathrm{mg}$ twice per day.

Over the course of the more than 3-yr data collection period, only one patient was lost to follow-up (table 2). Mean followup was $2.1 \pm 0.5 \mathrm{yrs}$, and 16 patients were followed for $>3 \mathrm{yrs}$. Recorded events included 19 deaths and three lung transplantations; no atrial septostomies were performed. To be conservative, the one patient lost to follow-up was considered dead at the last contact, bringing the number of deaths in the analyses to 20. Similarly, this patient was also considered to have received an alternate therapy at the last contact, as were three patients for whom treatment was unknown during some period. Thus, a total of 39 patients (including the three for whom treatment was unknown and the one who was lost to follow up) were considered to have received a prostanoid 


\begin{tabular}{|c|c|c|}
\hline & $\begin{array}{l}\text { Bosentan-treated } \\
\text { PPH patients }\end{array}$ & $\begin{array}{c}\text { NIH Registry PPH } \\
\text { patients }^{\#}\end{array}$ \\
\hline Subjects $n$ & 169 & 187 \\
\hline Sex male/female \% & $21 / 79$ & $37 / 63$ \\
\hline \multicolumn{3}{|l|}{ Age yrs } \\
\hline Mean \pm SD & $46 \pm 16$ & $36 \pm 15$ \\
\hline Range & $13-80$ & $1-81$ \\
\hline \multicolumn{3}{|l|}{ WHO functional class $\%$} \\
\hline$|/| I$ & $1 / 8^{\circ}$ & $0 / 29$ \\
\hline III/IV & $82 / 9$ & 71 \\
\hline \multicolumn{3}{|c|}{ Time from diagnosis months ${ }^{+}$} \\
\hline Mean \pm SD & $32 \pm 41$ & \\
\hline Range & $0.3-326$ & \\
\hline \multicolumn{3}{|l|}{ Haemodynamics mean $\pm S^{+}$} \\
\hline Cardiac index $\mathrm{L} \cdot \mathrm{min}^{-1} \cdot \mathrm{m}^{-2}$ & $2.35 \pm 0.80$ & $2.3 \pm 0.9$ \\
\hline PVR Wood units & $12.9 \pm 8.4$ & \\
\hline mPAP mmHg & $57.1 \pm 16.0$ & $60 \pm 18$ \\
\hline mRAP mmHg & $10.1 \pm 5.9$ & $9.7 \pm 6.3$ \\
\hline \multicolumn{3}{|l|}{ Walk test $\mathbf{m}$} \\
\hline Mean $\pm S D$ & $345 \pm 87$ & \\
\hline
\end{tabular}

PPH: primary pulmonary hypertension; NIH: National Institutes of Health; WHO: World Health Organization; PVR: pulmonary vascular pressure; mPAP: mean pulmonary arterial pressure; mRAP: mean right atrial pressure. \#: data from RICH et al. [10] provided for context, NIH Registry data were not directly used in any analysis; ": all patients entered the pivotal studies in functional class III or IV, but 15 (9\%) patients in the placebo group improved before being switched to bosentan in the extension study; ${ }^{+}$: time from diagnosis and haemodynamic data were available for 157-169 bosentan-treated patients, and for most of these patients were available only at the start of the placebo-controlled study.

derivative (epoprostenol, iloprost, treprostinil) or alternative oral therapy (sitaxsentan, sildenafil) at some time, either instead of or in addition to bosentan. Outcome categories were not mutually exclusive, and patients may have received more than one alternative therapy.

The Kaplan-Meier survival estimate at 2 yrs was $89 \%$ in $\mathrm{PPH}$ patients given first-line bosentan therapy (fig. 1, table 3). In contrast, the predicted 2-yr survival of these patients based on the $\mathrm{NIH}$ equation was $57 \%$. At each 6-month interval, observed survival was significantly better than predicted, as indicated by the $99.9 \%$ confidence intervals of the Kaplan-Meier estimates. Overall, patients receiving first-line bosentan therapy had a $5.5 \%$ annual death rate. Sixteen patients were followed for more than the 36 months covered in the analysis, and none had died as of the cut-off date.

Bosentan was used as the first arm of a treatment strategy in this study. While bosentan therapy was given first line, other treatments may have been administered over the course of the observation period. However, after 12 and 24 months of follow-up, $85 \%$ and $70 \%$ of patients, respectively, remained on bosentan monotherapy (fig. 2). Another 7\% at both 12 and 24 months continued bosentan while receiving additional therapy. Of the initial cohort, $78 \%$ and $55 \%$ were alive and on bosentan monotherapy at 1 and 2 yrs, respectively.

\section{TABLE 2 Outcomes}

Bosentan-treated PPH patients

\begin{tabular}{|c|c|}
\hline Subjects $n$ & 169 \\
\hline \multicolumn{2}{|l|}{ Duration of observation for survival } \\
\hline Mean $\pm S D$ & $2.1 \pm 0.5 \mathrm{yrs}$ \\
\hline Range & $0.1-3.3 \mathrm{yrs}$ \\
\hline Patients lost to follow-up & 1 \\
\hline Lung transplantations & 3 \\
\hline Deaths n (\%) & $20(12)^{\#}$ \\
\hline $\begin{array}{l}\text { Transfers to/additions of prostanoids or alternative } \\
\text { oral PPH therapies } \mathbf{n}(\%)\end{array}$ & $39(23)^{4}$ \\
\hline $\begin{array}{l}\text { Patients who received alternative treatment } \\
\text { in addition to bosentan }\end{array}$ & 19 \\
\hline $\begin{array}{l}\text { Discontinuations of bosentan without other event or } \\
\text { treatment }\end{array}$ & 4 \\
\hline
\end{tabular}

Baseline parameters that might be prognostic of survival were explored in a post hoc subgroup analysis (table 4). Both the magnitude of the hazard ratios and the $95 \%$ confidence limits that did not cross 1.0 suggest that baseline functional class and walk distance were associated with a poor outcome. While none of the haemodynamic variables studied were a statistically significant predictor of survival, the trend towards a poor outcome was most relevant for right atrial pressure above the median. Low cardiac index and low pulmonary artery pressure were more weakly correlated with a poor outcome.

Adverse events were collected systematically during the placebo-controlled trials and their open-label extension trials. Once bosentan became commercially available, patients were

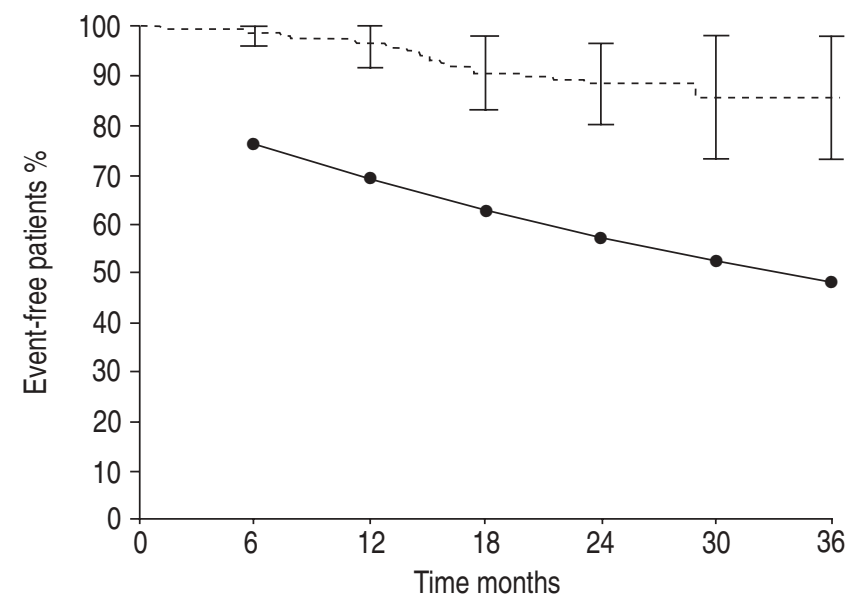

FIGURE 1. Kaplan-Meier estimates with $99.9 \%$ confidence intervals and predicted survival using the D'Alonzo equation [9]. The $99.9 \%$ confidence intervals of the Kaplan-Meier estimates do not approach the predicted survival, demonstrating a significant difference between the two curves. ----: observed survival; - : predicted survival. 
TABLE 3 Kaplan-Meier (K-M) estimates and predicted survival using the D'Alonzo equation [9]

\begin{tabular}{lccccccc} 
Time months & $\mathbf{0}$ & $\mathbf{6}$ & $\mathbf{1 2}$ & $\mathbf{1 8}$ & $\mathbf{2 4}$ & $\mathbf{3 0}$ & $\mathbf{3 6}$ \\
\hline K-M estimates \% & 100 & 98.8 & 96.4 & 90.5 & 88.5 & 85.6 & 85.6 \\
Predicted survival \% & & 76.3 & 69.2 & 62.8 & 57.3 & 52.4 & 48.2 \\
Patients at risk $\mathbf{n}$ & 169 & 167 & 163 & 153 & 113 & 23 & 16 \\
\hline
\end{tabular}

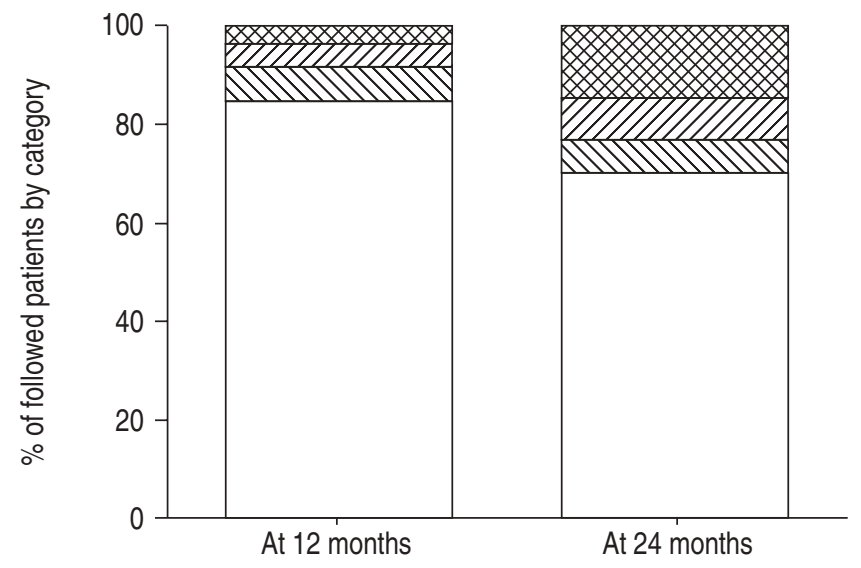

FIGURE 2. Vital status and treatment at 12 and 24 months of follow-up. All 169 patients were followed for 12 months; 132 patients were followed for at least 24 months. Percentages are based on the number of patients followed. $\mathbf{m}$ : dead; $\mathbb{Z}$ : alive, not on bosentan; $\mathbb{\mathbb { N }}$ : alive, on bosentan and other treatments; $\square$ : alive, on bosentan alone.

transitioned to commercial drug, were no longer considered to be in either of the studies, and were not systematically followed by the sponsor for adverse events. The mean duration of observation for adverse events as part of the placebocontrolled and open-label extension trials was $78 \pm 28$ weeks $( \pm S D)$ with a median of 77 weeks. During this observation period, $14.9 \%$ of patients had an elevation in hepatic transaminases to greater than three times the upper limit of normal, including $3.0 \%$ to greater than five to eight times the upper limit of normal and $4.2 \%$ to greater than eight times the upper limit of normal. The Kaplan-Meier curve estimating

\begin{tabular}{lccc} 
TABLE 4 & Prognostic factors for mortality & \\
& $\begin{array}{c}\text { Dichotomy/ } \\
\text { median }\end{array}$ & $\begin{array}{c}\text { Hazard } \\
\text { ratio }\end{array}$ & $\begin{array}{c}\mathbf{9 5 \%} \text { Confidence } \\
\text { limits }\end{array}$ \\
\hline Subjects $\mathbf{n}$ & 169 & & \\
WHO functional class & IV & 3.2 & $1.1-9.7$ \\
Cardiac index L· min. $\mathbf{m}^{-2}$ & $\leqslant 2.25$ & 1.5 & $0.6-3.6$ \\
mPAP $\mathbf{m m H g}$ & $\leqslant 54$ & 1.4 & $0.6-3.6$ \\
mRAP $\mathbf{m m H g}$ & $>8.5$ & 1.9 & $0.7-4.8$ \\
6MWT $\mathbf{m}$ & $\leqslant 358$ & 4.0 & $1.3-12.2$ \\
\hline
\end{tabular}

WHO: World Health Organization; mPAP: mean pulmonary arterial pressure; mRAP: mean right atrial pressure; $6 \mathrm{MWT}$ : 6-min walk test. time to first elevation of hepatic transaminases to greater than three times the upper limit of normal is displayed in figure 3. Adverse events that occurred with a frequency of $>10 \%$ during this observation period are listed in table 5 .

\section{DISCUSSION}

The treatment of PPH has realised dramatic advances over the past decade. At the time of the NIH Registry in the 1980s, there were no Food and Drug Administration (FDA) approved therapies for PPH. Currently, there are three therapies that have been FDA approved for PPH, and more are being studied. Due to the high mortality of $\mathrm{PPH}$, most controlled studies performed for the purposes of drug registration have been of 12-16 weeks duration, as investigators have been unwilling to randomise patients to placebo for a longer period. However, the wisdom of this practice has been called into question based on the results of a recent trial evaluating beraprost therapy for PAH [12]. In what was the longest placebo-controlled trial in $\mathrm{PAH}$ to date, an improvement in 6MWD was demonstrated at 3 and 6 months, however, the treatment benefit did not persist at 9 and 12 months. Although it may not be ethically feasible to conduct long-term, placebocontrolled trials of $\mathrm{PAH}$ therapies, it is imperative that the long-term effectiveness of such therapies are studied.

Epoprostenol was FDA approved for the treatment of PPH in 1995 based on the results of an 81-patient study [3]. The primary endpoint of 6MWD improved in patients treated with epoprostenol plus conventional therapy versus those treated with conventional therapy alone, however, a mortality benefit was also observed. Notably, all eight patients who died during the 12-week study period were randomised to conventional therapy alone. These eight patients were also of particularly high risk, with baseline $6 \mathrm{MWD}$ of $<150 \mathrm{~m}$. It was not until 7 yrs later that the long-term survival benefit of epoprostenol in PPH patients was demonstrated in two large series [4, 5]. Although life saving, epoprostenol also has potentially

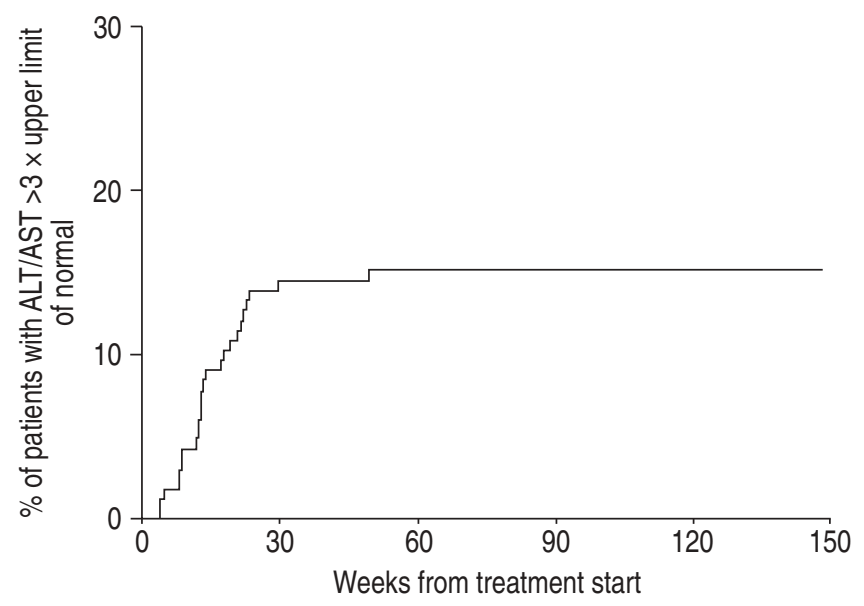

FIGURE 3. Kaplan-Meier estimates of time to the first elevation of hepatic transaminases to greater than three times the upper limit of normal. About $90 \%$ of the cases of elevated liver transaminases occurred during the first 26 weeks of treatment. ALT/AST: alanine aminotransferase/aspartate aminotransferase. Patients at risk: $168,139,111,50,11,0$ at $0,30,60,90,120$ and 150 weeks from treatment start, respectively. 


\begin{tabular}{lcc}
\hline TABLE 5 Adverse events noted by $>10 \%$ of patients \\
\hline Body system/adverse events & $\mathbf{n}$ & $\%$ \\
\hline & & \\
Aggravated pulmonary hypertension & 56 & 33.1 \\
Headache & 53 & 31.4 \\
Upper respiratory tract infection & 36 & 21.3 \\
Chest pain & 32 & 18.9 \\
Dizziness (except vertigo) & 29 & 17.2 \\
Dyspnoea & 27 & 16.0 \\
Flushing & 24 & 14.2 \\
Nasopharyngitis & 24 & 14.2 \\
Diarrhoea & 23 & 13.6 \\
Syncope & 23 & 13.6 \\
Abnormal hepatic function & 22 & 13.0 \\
Bronchitis & 20 & 11.8 \\
Nausea & 20 & 11.8 \\
Cardiac failure & 19 & 11.2 \\
Leg oedema & 19 & 11.2 \\
Cough & 18 & 10.7 \\
Pain in limb & 18 & 10.7 \\
Arthralgia & 17 & 10.1 \\
Palpitations & 17 & 10.1 \\
\hline
\end{tabular}

Adverse events were collected from the 169 patients during adverse event reporting periods only. The mean duration of observation for adverse events was $78 \pm 28$ weeks ( $\pm S D$, median 77 weeks).

life-threatening adverse effects, with deaths related to infection and infusion interruption.

The mortality benefit of epoprostenol in $\mathrm{PPH}$ has prevented other investigational therapies from demonstrating a survival benefit during a 12- to 16-week placebo-controlled trial for two reasons: first, the most critically ill patients, those who would be most likely to die over this short period of time, are generally not enrolled in placebo-controlled trials. They are frequently treated with epoprostenol, as it is considered unethical to enrol such a patient in a placebo-controlled trial. Secondly, if a patient deteriorates during a placebo-controlled trial, they are often withdrawn from the trial and treated with epoprostenol. As a result, longer-term observational studies are required to demonstrate a survival benefit of newer agents.

The present study demonstrates, in a long-term observational manner, that first-line therapy with the dual endothelin receptor antagonist bosentan, followed by addition of other therapy if needed, improves survival in $\mathrm{PPH}$ patients. Patients who were enrolled in the two placebo-controlled trials of 12-28 weeks duration were followed for a mean of $2.1 \pm 0.5 \mathrm{yrs}$. The survival at 2 yrs was $89 \%$ compared to a predicted survival of $57 \%$ based on the equation formulated from the NIH Registry. The NIH Registry predicted survival was used in the present study as it is not possible to follow a contemporary patient population without therapy. Those patients with more advanced symptoms (functional class IV) and lower exercise tolerance (6MWD below the median of $358 \mathrm{~m}$ ) had a worse outcome with first-line bosentan therapy, similar to what has been demonstrated in the natural history of $\mathrm{PPH}$ and in epoprostenol-treated $\mathrm{PPH}$ patients [4, 5]. The number of functional class IV patients in the current study was small. Given this small sample size, the higher mortality, and the more rapid onset of beneficial effects of epoprostenol (compared with bosentan), it may be appropriate to treat functional class IV patients with epoprostenol, at least initially.

Interestingly, of the prognostic variables studied by univariate analysis, the two noninvasive parameters of functional class and $6 \mathrm{MWD}$ were the most powerful predictors of outcome. The prognostic value of functional class has been noted in both untreated PPH patients in the NIH Registry, and in $\mathrm{PPH}$ patients treated with epoprostenol $[4,5,10]$. Exercise endurance has also been previously correlated with outcome in epoprostenol-treated PPH patients [3-5]. While none of the invasive haemodynamic variables reached statistical significance, the strongest trend was noted for right atrial pressure. The unexpected trend towards a poor outcome with a baseline mean pulmonary artery pressure below the median mirrors a previous observation in epoprostenol-treated $\mathrm{PPH}$ patients [4]. This suggests that mean pulmonary artery pressure may not be the most direct indicator of disease severity, and treatment outcome might be better assessed using other indicators of right ventricular function, such as cardiac index and right atrial pressure, both at baseline and on treatment. Other variables that may have prognostic value in $\mathrm{PPH}$, such as troponin, brain natriuretic peptide, uric acid, and echocardiographic variables were not systematically collected during the placebo-controlled trials and, thus, were not analysed as prognostic variables in the current study.

The favourable safety profile documented in the two placebocontrolled trials appears to persist over the long term. While the incidence of hepatic transaminase elevation was slightly greater than that observed over the 12- to 16-week placebocontrolled trials, there were few new cases after the first 26 weeks on treatment and no serious, irreversible hepatic dysfunction was reported. It is important to note that nearly half $(7.2 \%)$ of the reported $14.9 \%$ of patients with liver function testing abnormalities had elevations in transaminases of greater than five times the upper limit of normal. The importance of following patients monthly for this potentially serious adverse event cannot be overemphasised. Other adverse events noted have commonly been reported in this particularly ill patient population. It should be noted that the length of follow-up for safety was less than that for survival (means of 1.5 and 2.1 yrs, respectively) and thus underestimates the frequency of adverse experiences over the entire follow-up period.

The main limitation of the current study is the use of the NIH Registry equation in lieu of a placebo or historical control group. The NIH equation is based on data from the 1980s, and background practice patterns, particularly the widespread use of oral anticoagulation, may have changed since then. However, studies demonstrating a survival benefit with oral anticoagulation have not been performed in a prospective and controlled matter. In fact, a recent evidence-based review only gave a moderate recommendation for the use of oral anticoagulation in patients with PPH [13]. Although the method used in the validation of the NIH equation was crude by later standards [14, 15], more recently published estimates of survival in PPH remain similar to the expected survival based 
on the NIH equation. In an evidence-based literature review conducted via a search of the Medline bibliographic database from 1992 to October 2002, 12-21 studies were identified that described 1, 2, or 3-yr survival of patients with $\mathrm{PPH}$ [16]. Unweighted and unadjusted averages of the entire data set demonstrated a survival of $79 \%$ at $1 \mathrm{yr}, 66 \%$ at $2 \mathrm{yrs}$, and $59 \%$ at 3 yrs. The same evidence-based review also described survival curves for PPH patients treated with conventional medical therapy versus epoprostenol during that time period. The 1, 2, and 3-yr survival was $72 \%, 53 \%$, and $48 \%$, respectively, in those patients who did not receive epoprostenol versus $82 \%, 74 \%$, and $62 \%$, respectively, in those patients who did receive epoprostenol. The reported survival in patients treated with conventional therapy is similar to the estimated survival based on the NIH equation that is used in the current study. Another limitation of this study is that patients were initially selected for participation in a placebocontrolled trial. Certainly, patients who participate in trials may not reflect the patients that clinicians most commonly see on an everyday basis.

Given the progressive nature of $\mathrm{PPH}$, it is unethical to perform a placebo-controlled trial of $2-3$ yrs duration. Additionally, during the extension studies other assessments of disease severity, such as 6MWD, haemodynamics, and functional classification were not systematically collected. It would have also been interesting to have followed other prognostic variables, such as brain natriuretic peptide or troponin. Documentation of such results could have substantially strengthened the results of the current study. Practice variations are another limitation. During the open-label extensions following the placebo-controlled trials, treatment patterns were not mandated by protocol and were left to the discretion of the investigator. It is important to note that these results are not those of bosentan therapy alone, but rather of a treatment strategy starting with bosentan. At the discretion of the investigator, a prostanoid or other therapy for $\mathrm{PPH}$ could have been added to or substituted for bosentan, after the placebo-controlled portion of the study. However, this reflects current variability in practice patterns and is a practical approach to the therapy of $\mathrm{PPH}$.

In conclusion, the present study suggests that the treatment of first-line bosentan therapy, followed by the addition of other pulmonary arterial hypertension therapies if needed, improves survival in patients with advanced primary pulmonary hypertension. Given the ease of administration and favourable side-effect profile, the strategy of treatment with first-line bosentan should be considered for World Health Organization functional class III primary pulmonary hypertension patients.

\section{ACKNOWLEDGEMENTS}

The authors thank the principal investigators of the studies and their staff: A. Keogh, Australia; M. Delcroix, Belgium; F. Kleber, Germany; I. Ben-Dov, Israel; T. Pulido, Mexico; A. Torbicki, Poland; M. Gomez-Sanchez, Spain; A.J. Peacock, J. Pepke-Zaba, UK; R. Channick, B. DeBoisblanc, T. DeMarco, R. Doyle, R. Frantz, A. Frost, M. Landzberg, S. Murali, R. Oudiz, I. Robbins, R. Schilz, V. Tapson, USA.

\section{REFERENCES}

1 Fuster V, Steele PM, Edwards WD. Primary pulmonary hypertension: natural history and the importance of thrombosis. Circulation 1984; 70: 580-587.

2 Rich S, Kaufmann E, Levy PS. The effect of high doses of calcium-channel blockers on survival of primary pulmonary hypertension. N Engl J Med 1992; 327: 76-81.

3 Barst RJ, Rubin LJ, Long WA, et al. A comparison of continuous intravenous epoprostenol (prostacyclin) with conventional therapy for primary pulmonary hypertension. New Engl J Med 1996; 334: 296-301.

4 Sitbon O, Humbert M, Nunes $\mathrm{H}$, et al. Long-term intravenous epoprostenol infusion in primary pulmonary hypertension: prognostic factors and survival. J Am Coll Cardiol 2002; 40: 780-788.

5 McLaughlin VV, Shillington A, Rich S. Survival in primary pulmonary hypertension: the impact of epoprostenol therapy. Circulation 2002; 106: 1477-1482.

6 Channick RN, Simonneau G, Sitbon O, et al. Effects of the dual endothelin-receptor antagonist bosentan in patients with pulmonary hypertension: a randomised placebocontrolled study. Lancet 2001; 358: 1119-1123.

7 Rubin LJ, Badesch DB, Barst RJ, et al. Bosentan therapy for pulmonary arterial hypertension. N Engl J Med 2002; 346: 896-903.

8 Sitbon O, Badesch DB, Channick RN, et al. Effects of the dual endothelin receptor antagonist bosentan in patients with pulmonary arterial hypertension. A 1-year follow-up study. Chest 2003; 124: 247-254.

9 D'Alonzo GE, Barst RJ, Ayres SM, et al. Survival in patients with primary pulmonary hypertension. Results from a national prospective registry. Ann Intern Med 1991; 115: 343-349.

10 Rich S, Dantzker DR, Ayres SM, et al. Primary pulmonary hypertension. A national prospective study. Ann Intern Med 1987; 107: 216-223.

11 Sandoval J, Bauerle O, Palomar A, et al. Survival in primary pulmonary hypertension. Validation of a prognostic equation. Circulation 1994; 89: 1733-1744.

12 Barst RJ, McGoon M, McLaughlin VV, et al. Beraprost therapy for pulmonary arterial hypertension. J Am Coll Cardiol 2003; 41: 2119-2125.

13 Badesch DB, Abman SN, Ahearn G, et al. Medical therapy for pulmonary arterial hypertension: ACCP evidencebased guidelines for clinical practice. Chest 2004; 126: Suppl. 1, 35S-77S.

14 Harrell Jr FE, Lee KL, Mark DB. Multivariable prognostic models: issues in developing models, evaluating assumptions and adequacy, and measuring and reducing errors. Stat Med 1996; 15: 361-387.

15 McGinn TG, Guyatt GH, Wyer PC, Naylor CD, Stiell IG, Richardson WS. Users' guides to the medical literature: XXII: how to use articles about clinical decision rules. Evidencebased Medicine Working Group. JAMA 2000; 284: 79-84.

16 McLaughlin VV, Presberg KW, Doyle RL, et al. Prognosis of pulmonary arterial hypertension: ACCP evidence-based clinical practice guidelines. Chest 2004; 126: Suppl. 1, 78S-92S. 